\title{
Identification of a Novel Exonic Mutation at -13 from 5' Splice Site Causing Exon Skipping in a Girl with Mitochondrial Acetoacetyl-Coenzyme A Thiolase Deficiency
}

\author{
Toshiyuki Fukao, * Seiji Yamaguchi, * \\ ${ }^{*}$ Department of Pediatrics, Gifu University School of Medicine, Gifu, Gifu 500, Japan; ${ }^{\ddagger}$ Department of Pediatrics, Shimane Medical \\ University, Izumo, Shimane 693, Japan; ${ }^{\S}$ Department of Pediatrics/Genetics, The University of Illinois at Chicago, Chicago, Illinois \\ 60612; and "Department of Biochemistry, Shinshu University School of Medicine, Matsumoto, Nagano 390, Japan
}

\begin{abstract}
We identified a novel exonic mutation which causes exon skipping in the mitochondrial acetoacetyl-CoA thiolase (T2) gene from a girl with T2 deficiency (GK07). GK07 is a compound heterozygote; the maternal allele has a novel $G$ to $T$ transversion at position 1136 causing Gly ${ }^{379}$ to Val substitution (G379V) of the $T 2$ precursor. In case of in vivo expression analysis, cells transfected with this mutant cDNA showed no evidence of restored $\mathrm{T} 2$ activity. The paternal allele was associated with exon 8 skipping at the cDNA level. At the gene level, a $C$ to $T$ transition causing $G \ln ^{272}$ to termination codon (Q272STOP) was identified within exon 8, 13 bp from the 5' splice site of intron 8 in the paternal allele. The mRNA with Q272STOP could not be detected in GK07 fibroblasts, presumably because pre-mRNA with Q272STOP was unstable because of the premature termination. In vivo splicing experiments revealed that the exonic mutation caused partial skipping of exon 8. This substitution was thought to alter the secondary structure of T2 pre-mRNA around exon 8 and thus impede normal splicing. The role of exon sequences in the splicing mechanism is indicated by the exon skipping which occurred with an exonic mutation. (J. Clin. Invest. 1994. 93:1035-1041.) Key words: $\beta$-ketothiolase deficiency $\bullet$ exon skipping • molecular basis • in vivo splicing experiment • organic aciduria
\end{abstract}

\section{Introduction}

Mitochondrial acetoacetyl-CoA thiolase (T2, ${ }^{1}$ EC 2.3.1.9) deficiency ( $\beta$-ketothiolase deficiency, 2 -methylacetoacetic aciduria, McKusick 203750) is an inborn error of isoleucine and ketone body catabolism characterized by intermittent ketoacidotic episodes and urinary excretion of 2-methylacetoacetate, 2-methyl-3-hydroxybutyrate, and tiglylglycine (1-4). Fibroblasts from one patient with this disorder lacked the $\mathrm{T} 2$ protein because of a defect in its biosynthesis (5). However, there is

Address correspondence to Dr. T. Fukao, Department of Pediatrics, Gifu University School of Medicine, 40 Tsukasa-machi, Gifu 500, Japan.

Received for publication 22 February 1993 and in revised form 1 November 1993.

1. Abbreviations used in this paper: RT-PCR, reverse transcription and PCR; T2, mitochondrial acetoacetyl-CoA thiolase.

J. Clin. Invest.

(C) The American Society for Clinical Investigation, Inc.

$0021-9738 / 94 / 03 / 1035 / 07 \quad \$ 2.00$

Volume 93, March 1994, 1035-1041 molecular heterogeneity in defects of T2 protein (6). Human T2 cDNA is about $1.5 \mathrm{~kb}$ and encodes the 424-amino acid T2 precursor (7). The 27-kb human T2 gene, with 12 exons and 11 introns (8), is located on chromosome 11q22.3-q23.1 (9). In five different families, we identified seven gene mutations responsible for this disease (10-13). Among them, four mutations at $5^{\prime}$ or $3^{\prime}$ splice sites caused skipping $(11,12)$ or aberrant splicing (13) of the corresponding exon. Conservation of nucleotide sequences of $5^{\prime}$ and $3^{\prime}$ splice sites and the branch site are critical for accurate splicing. However, these events alone cannot account for accuracy of the splicing. Recent studies suggest that exonic sequences and a secondary structure of the RNA precursor have essential information for accurate splice site selection (14-18).

We describe herein the molecular basis of $\mathrm{T} 2$ deficiency in a 3-yr-old girl (GK07). In one of her mutant alleles, a novel mutation within exon 8 resulted in partial skipping of exon 8 . We clarified this phenomenon in in vivo splicing experiments.

\section{Methods}

Case. GK07 is a Caucasian girl born in 1986 to nonconsanguineous parents. The diagnosis of T2 deficiency was made when she was $3 \mathrm{yr}$ old and after multiple ketoacidotic attacks. The patient's growth and development were normal, and there was no mental retardation.

Protein analyses. Fibroblasts from GK07 and her parents were cultured in Eagle's MEM containing 10\% FCS. Enzyme assay, immunoblot analysis, and pulse-chase experiments were done, as described elsewhere $(5,11)$.

Reverse transcription and $P C R(R T-P C R)$ and genomic DNA analyses. Oligonucleotide primers for RT-PCR and genomic DNA amplification around exon 8 were performed as described (11). RT-PCR and DNA sequence analysis of products subcloned into plasmid were done, as described elsewhere (11).

In vivo expression of T2 cDNAs. Transient expression of T2 cDNAs was performed as described elsewhere (19). Briefly, full length normal and mutant cDNAs were subcloned separately into an in vivo expression vector, pCAGGS (20). $2 \mu \mathrm{g}$ of plasmid was transfected with TransfectAce (GIBCO BRL, Gaithersburg, MD) into $5 \times 10^{5}$ SV40transformed fibroblasts from GK03, a T2-deficient patient in whom T2 mRNA was strikingly decreased (7). Cells were harvested $72 \mathrm{~h}$ after transfection and were assayed for T2 activity.

Secondary structure predictions. Normal and mutant sequences of exon 8 with dinucleotides of the 5 ' and 3 ' splice sites were analyzed by Zuker and Stiegler's method (21) using DNASIS-MAC software (Hitachi Software Engineering Co., Yokohama, Japan).

In vivo splicing experiments. Details of the methods are given in the legend to Fig. 7. Primers used are as follows, and underlined nucleotides are mismatches or linker sequences:

A primer on exon 7 sequence (5'-AGCTGTGCTGAGAATTCAGC-3');

B primer on exon 9 sequence (3'-TTACGGTCATGTGACTTAAGACC-5'); 


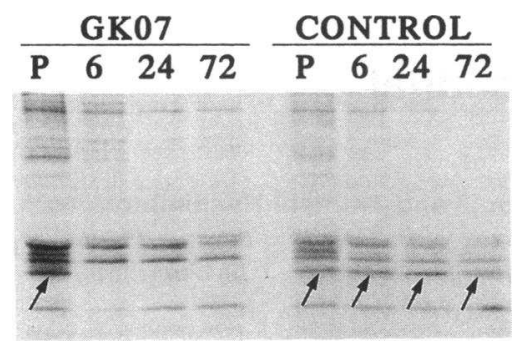

Figure 1. Pulse-chase experiments of fibroblasts. Fibroblasts from patient GK07 and a control individual were pulse labeled with Tran ${ }^{35}$ S-label (ICN Biomedicals, Inc., Costa Mesa, $\mathrm{CA}$ ) for $1 \mathrm{~h}$ and were chased for 6,24 , and 72 h. Cell extracts were immunoprecipitated with anti-rat T2 IgG and were applied to SDSPAGE. In control lanes, one-third the amount of immunoprecipitates was applied. Arrows indicate T2 subunits.

C primer on exon 7 sequence (5'-TACCAGAAGTAAAGCAGCAT-3'); and

D primer on rabbit $\beta$-globin sequence ( $3^{\prime}$-GTCCGTGTGTTTATGGTG-5')

Rabbit $\beta$-globin primer sequence was obtained as described (22).

Quantitative estimation of PCR products. Estimation of the ratio of mRNA species with/without exon 8 sequence was made as follows. Two plasmids containing cDNA fragments with and without exon 8 sequence were diluted to fg order. The plasmids were mixed with resulting ratios of 10:1, 10:2,10:3,10:4, and 10:5 with/without exon 8 . PCR amplification was done using these mixed plasmids, the first strand cDNA generated from GK07 fibroblasts, and ${ }^{32} \mathrm{P}$-end-labeled primer in 15,20, and 25 cycles. Products were electrophoresed on a 5\% acrylamide gel followed by fluorography and densitometric analysis. The intensities of bands with/without exon 8 sequence from the cDNA pool were compared with those from the mixed plasmids of differencing ratios. The ratio of exon 8-plus/exon 8-minus mRNA species was determined to be that of the comparable mixed plasmid samples.

\section{Results}

Protein analyses. T2 enzyme activities of skin fibroblast extracts from patient GK07 and her parents are shown in Table I. Extract from GK07 was deficient in T2, as deduced from the failure of $\mathrm{K}^{+}$to increase acetoacetyl-CoA thiolase activity. T2 activities in the parents were consistent with heterozygosity for T2 deficiency with $+\mathrm{K}^{+} /-\mathrm{K}^{+}$values intermediate between
Table I. Thiolase Activity in Skin Fibroblasts

\begin{tabular}{lcccc}
\hline & \multicolumn{3}{c}{ Acetoacetyl-CoA } & \\
\cline { 2 - 4 } \multicolumn{1}{c}{ Substrate } & $+\mathrm{K}^{+}$ & $-\mathrm{K}^{+}$ & $+\mathrm{K}^{+} /-\mathrm{K}^{+}$ & 3-ketooctanoyl-CoA \\
\hline Patient GK07 & 4.6 & 4.6 & 1.0 & 8.0 \\
Father & 5.2 & 3.3 & 1.6 & 8.5 \\
Mother & 4.9 & 3.1 & 1.6 & 7.6 \\
Controls & 9.1 & 4.6 & 2.0 & 9.9 \\
$(n=10$ range) & $(6.8-11.9)$ & $(3.3-6.3)$ & $(1.8-2.1)$ & $(6.9-12.4)$ \\
& & & & \\
\hline
\end{tabular}

The acetoacetyl-CoA thiolase activity determined in the absence $\left(-K^{+}\right)$or in the presence $\left(+K^{+}\right)$and the ratio of these two activities $\left(+K^{+} /-K^{+}\right)$is listed. The activity with 3-ketooctanoyl-CoA was determined in the presence of $\mathrm{K}^{+}$. Enzyme activities were determined as milliunits per milligram of protein.

the patient and controls. Fibroblast extracts from patient GK07 and her parents showed levels of acetoacetyl-CoA $\left(-\mathrm{K}^{+}\right)$and 3-ketooctanoyl-CoA thiolase activities comparable with findings in the controls. Immunoblot analysis of fibroblast extracts revealed that $\mathrm{T} 2$ protein was absent in GK07 (data not shown). Fibroblast $\mathrm{T} 2$ protein was decreased in both parents, as compared with findings in control extracts (data not shown). Pulse-chase experiments of T2 protein are shown in Fig. 1. T2 protein of GK07 present at $1 \mathrm{~h}$ was not detectable with a 6-h chase, while that of the control was evident with a 72-h chase. Thus, T2 protein generated by GK07 was apparently unstable.

Identification of mutations in T2 cDNA from patient GKO7. Amplified cDNA fragments were analyzed by electrophoresis on a $2 \%$ agarose gel (Fig. $2 \mathrm{~A}$ ). A single band of the expected size was obtained in fragments $\mathrm{A}, \mathrm{B}$, and $\mathrm{C}$ from the patient's mother $(M)$. Amplification of cDNA from the patient GK07 $(P)$ and her father $(F)$ revealed an additional fragment smaller than that of the expected size by $100 \mathrm{bp}$ in fragments B and C (arrows). The amount of shorter fragments was about one-fifth that of the normal fragment, as estimated by densitometric analysis of these fragments amplified with ${ }^{32} \mathrm{P}$-labeled primers.

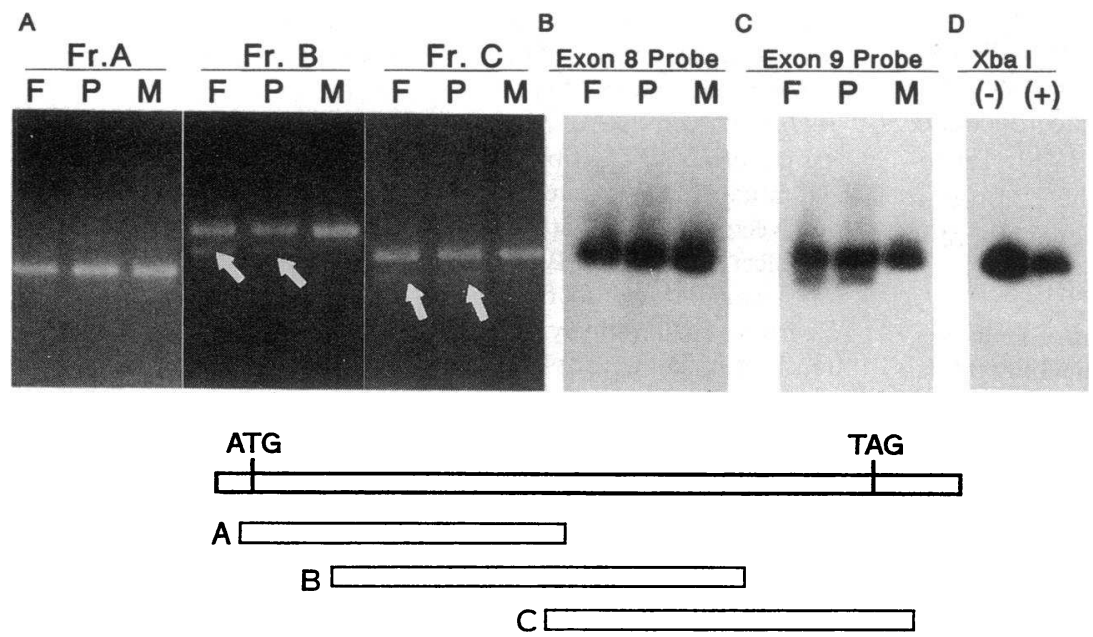

Figure 2. Amplified T2 cDNA fragments of patient GK07's family. $A$ shows electrophoresis on $2.0 \%$ agarose gel. The coding region of T2 cDNA was amplified in three separate fragments, A, B, and $\mathrm{C}$, as shown in the lower panel. $A T G$ and $T A G$ indicate positions of the initiator methionine codon and the termination codon of T2 cDNA, respectively. $M, P$, and $F$ indicate the mother, patient (GK07), and father, respectively. Arrows indicate the fragment which is $100 \mathrm{bp}$ smaller than that of the expected size. $B$ and $C$ show Southern blot analysis of amplified $C$ fragments with exon 8 and exon 9 probes, respectively. Exon 8 and exon 9 probes were obtained by genomic amplifications with In 7B [ 5'-ATTCTAGATGAGTGTTTACTTGG-3'] and In 8A [ 3'-GTCATATAGTCCGAGTACA-5'], and In 8B [ 5'-TGCAGTTAGCTGTGTACATG-3'] and In 9A [3'-ATCGACTCGTACCACCGTACTAGTA-5'], respectively, adding $\left[\alpha-{ }^{32} \mathrm{P}\right] \mathrm{dCTP}$ as substrate. $D$ shows a Southern blot of normal sized $C$ fragments from GK07 before $(-)$ and after $(+)$ Xbal digestion. If the $C$ fragment with the Q272STOP mutation was present in normal sized C fragment pool from GK07, 545- and 242-bp fragments should be generated. 
Two mutations were identified in fragments from GK07's cDNA fragments. The first was a point mutation, $G$ to $T$ at position 1136, encoding an amino acid change from $\mathrm{Gly}^{379}$ to Val of the T2 precursor (G379V) in the normal sized C fragment (Fig. 3 A, larger fr. ). This G379V mutation was detected
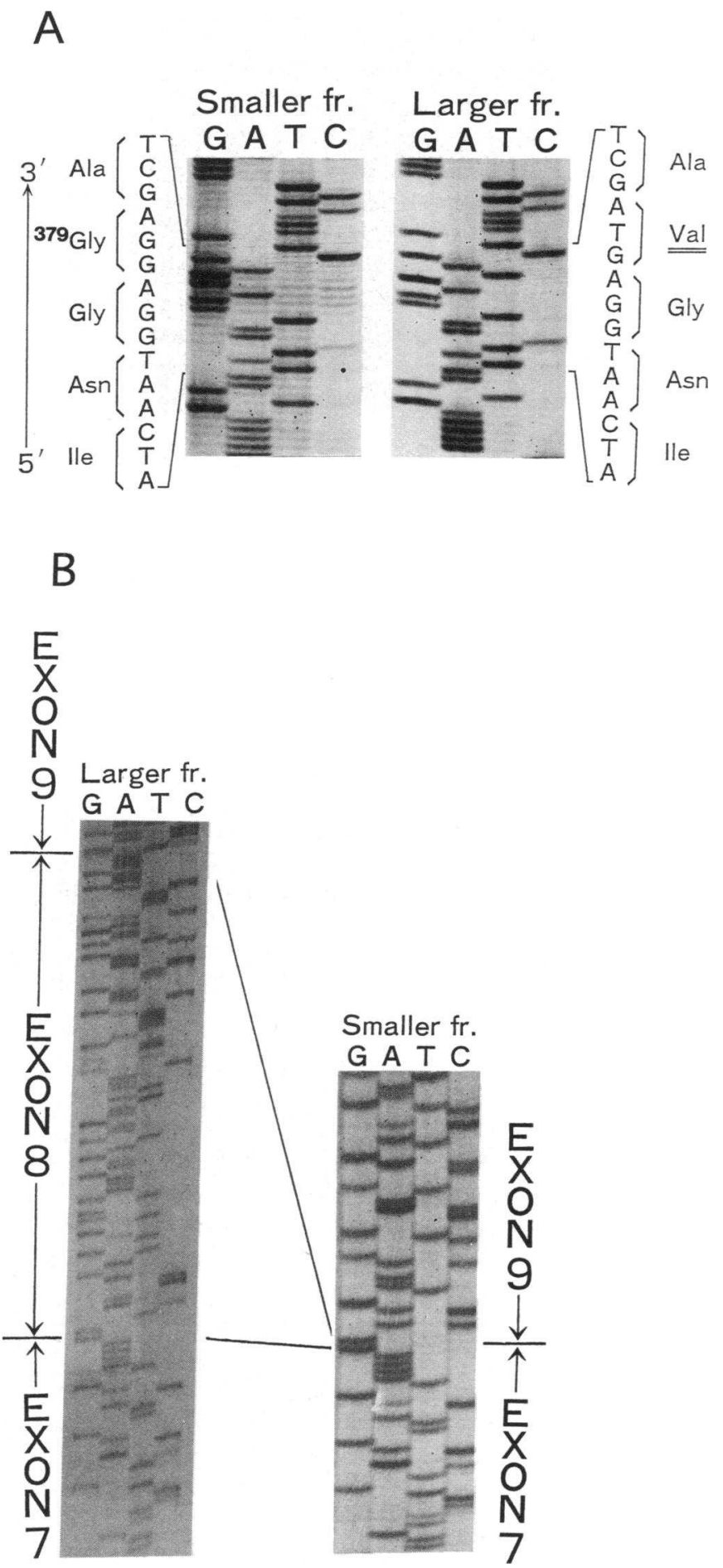

Figure 3. Partial nucleotide sequences of fragments $\mathrm{C}$. The mutated regions are shown. $(A) A$ single base substitution of $\mathrm{G}$ to $\mathrm{T}$ at position 1136 results in replacement of $\mathrm{Gly}^{379}$ (GGA) by Val (GTA) in the larger fragment $C$. $(B)$ Exon 8 skipping in the smaller fragment $C$ is shown.

Table II. Enzyme Assays after

In Vivo Transient Expression of T2 cDNAs

\begin{tabular}{llcc}
\hline & \multicolumn{3}{c}{ Acetoacetyl-CoA thiolase activities } \\
\cline { 2 - 4 } & $-\mathrm{K}^{+}$ & $+\mathrm{K}^{+}$ & $+\mathrm{K}^{+} /-\mathrm{K}^{+}$ \\
\hline pCAGGST2 (mock cDNA) & $16.0 \pm 2.2$ & $16.5 \pm 1.7$ & 1.1 \\
pCAGGST2 (normal) & $45.2 \pm 7.0$ & $164 \pm 44$ & 3.6 \\
pCAGGST2 (exon 8 deleted) & $19.3 \pm 3.8$ & $20.0 \pm 4.0$ & 1.0 \\
pCAGGST2 (G379V) & $16.0 \pm 0.81$ & $16.0 \pm 0.81$ & 1.0 \\
& & & \\
\hline
\end{tabular}

pCAGGST2s were transfected to SV40-transformed GK03's fibroblasts, and thiolase activities were assayed $72 \mathrm{~h}$ later.

in two of five clones of the maternal $\mathrm{C}$ fragments, hence this mutation located on exon 11 was inherited from the mother (data not shown). The second was a deletion of positions 731826 in the smaller B and C fragments. This deletion corresponds precisely to exon 8 , indicating that skipping of this exon had occurred (Fig. $3 \mathrm{~B}$ ). Because the smaller fragment with exon 8 deleted was present in amplified DNA from the father, but not from the mother of patient GK07, we considered this deletion to be derived from the paternal allele. Findings in Southern blot analysis of amplified $C$ fragments with exon 8 (Fig. $2 B$ ) and exon 9 (Fig. $2 C$ ) probes are in accord with the above results. No other mutation was identified in T2 cDNA from GK07.

Characterization of mutant T2 cDNAs. We reported that mutant T2 derived from the mRNA with exon 8 sequence deleted has no thiolase activity, as determined in in vivo transient expression assays (19). We constructed a full length cDNA with the G379V mutation and subcloned it into the transient expression vector, pCAGGS. The result of enzyme assay after in vivo expression is summarized in Table II. Activation of acetoacetyl-CoA thiolase activity by $\mathrm{K}^{+}$was nil in cells transfected with either pCAGGST2 (exon 8 deleted) or pCAGGST2 $(G 379 \mathrm{~V})$, whereas activation did occur in cells transfected with pCAGGST2 (normal). We concluded that these mutations are responsible for the T2 deficiency.

The G379V mutation is located in the carboxy-terminal region which is highly conserved among seven reported thiolases (23-29) (Fig. 4). In fibroblasts from patient GK07, unsta-

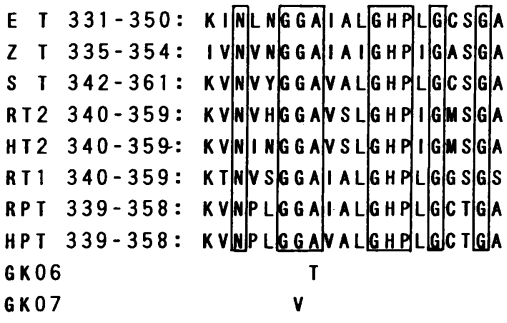
CoA thiolase of Zoogloea ramigera (26); ST, cytosolic acetoacetylCoA thiolase of Saccharomyces uvarum (27); RT2, rat T2 (28); HT2, human T2 (11); RT1, rat mitochondrial 3-ketoacyl-CoA thiolase (29); RPT, rat peroxisomal 3-ketoacyl-CoA thiolase (30); and HPT, human peroxisomal 3-ketoacyl-CoA thiolase (31). These sequences were aligned to achieve maximum homologies. Amino acid residues are numbered beginning at the amino-terminal residues of mature enzymes in the case of T2 and PT. Hence G379V and A380T mutations are positioned at 346 and 347, respectively. 
B
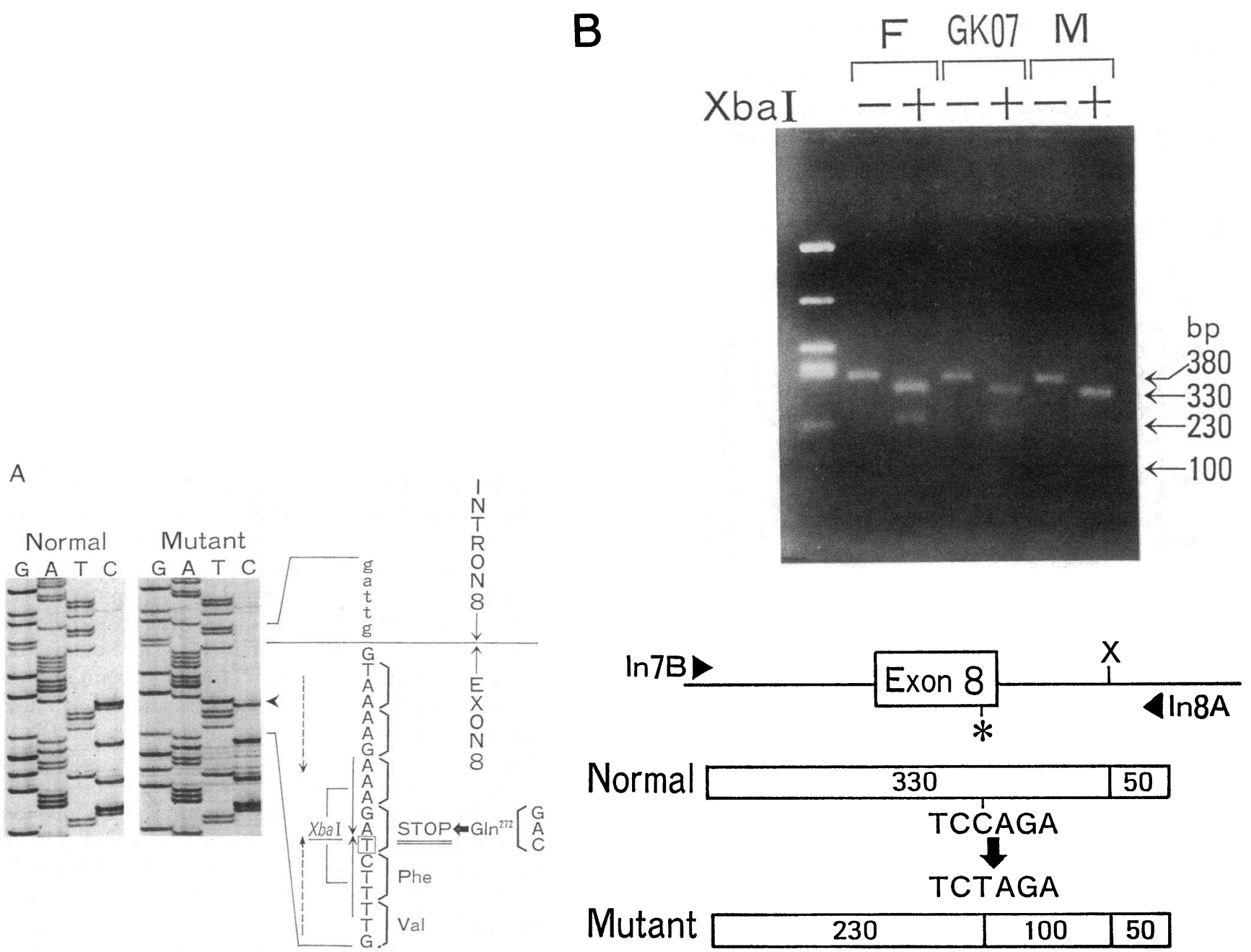

Figure 5. Identification of Q272STOP mutation in the exon 8 sequence. Genomic sequences around exon 8 were amplified with primers In 7B and In 8A. $A$ shows the partial nucleotide sequence around the 5' splice site of intron 8. A C to T mutation (Q272STOP) was identified (arrowhead). This mutation creates an $\mathrm{Xbal}$ site. Arrows show palindromic sequences enhanced by the mutation. $B$ demonstrates familial analysis with XbaI digestion. Fragments from the father (lane $F$ ) and GK07 were partially digested to 230- and 100-bp fragments, hence they are probably heterozygotes of Q272STOP mutation.

ble mutant T2 protein of the normal size was detected by pulse-chase experiments (Fig. 1). The mutant protein was likely derived from the T2 mRNA with G379V mutation because the other mutant allele would produce a 33-amino acidtruncated species by skipping of exon 8 . We noted in pulsechase experiments, after in vivo transient expression, that the A380T mutation detected in GK06, adjacent to the G379V mutation, made T2 protein unstable (10). This highly conserved region may be important for stability of thiolase proteins. The precise role of this region remains to be determined.

Identification of a point mutation in exon 8 associated with exon 8 skipping. The region around exon 8 of GK07's genomic DNA was amplified and sequenced. We found no evidence of a substitution in either the 3 ' splice site of intron 7 or the 5 ' splice site of intron 8 . However, there was a $\mathrm{C}$ to $\mathrm{T}$ mutation within exon 8 at position -13 from the $5^{\prime}$ splice site of intron 8 . This change altered the codon in amino acid 272 from glutamine to a termination codon (Q272STOP) and a new Xbal site was created on this mutant allele (Fig. $5 A$ ). Xbal restriction assay of the amplified genomic fragments (Fig. $5 B$ ) showed that the Q272STOP mutation was heterozygous in patient GK07 and
A NORMAL
B MUTANT

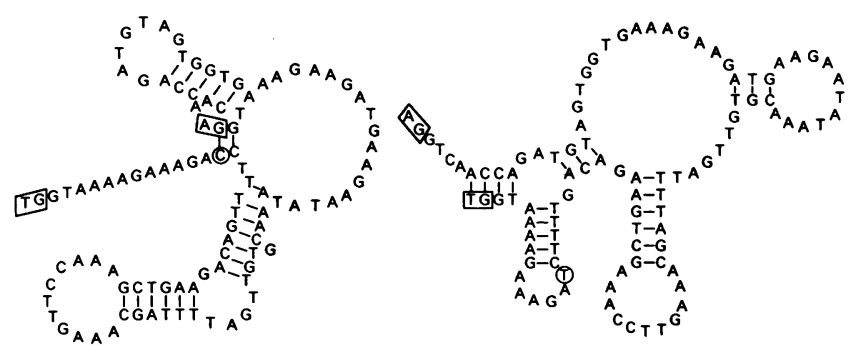

Figure 6. Prediction of pre-mRNA secondary structures around exon 8 . Secondary structures of normal and mutant sequences of exon 8 were predicted by Zuker and Stiegler's method (21). Boxed sequences are dinucleotides of the $5^{\prime}$ or $3^{\prime}$ splice site. Nucleotides encircled are sites of the Q272STOP mutation. $A$ shows normal sequence, and $B$ shows mutant sequence. 
A
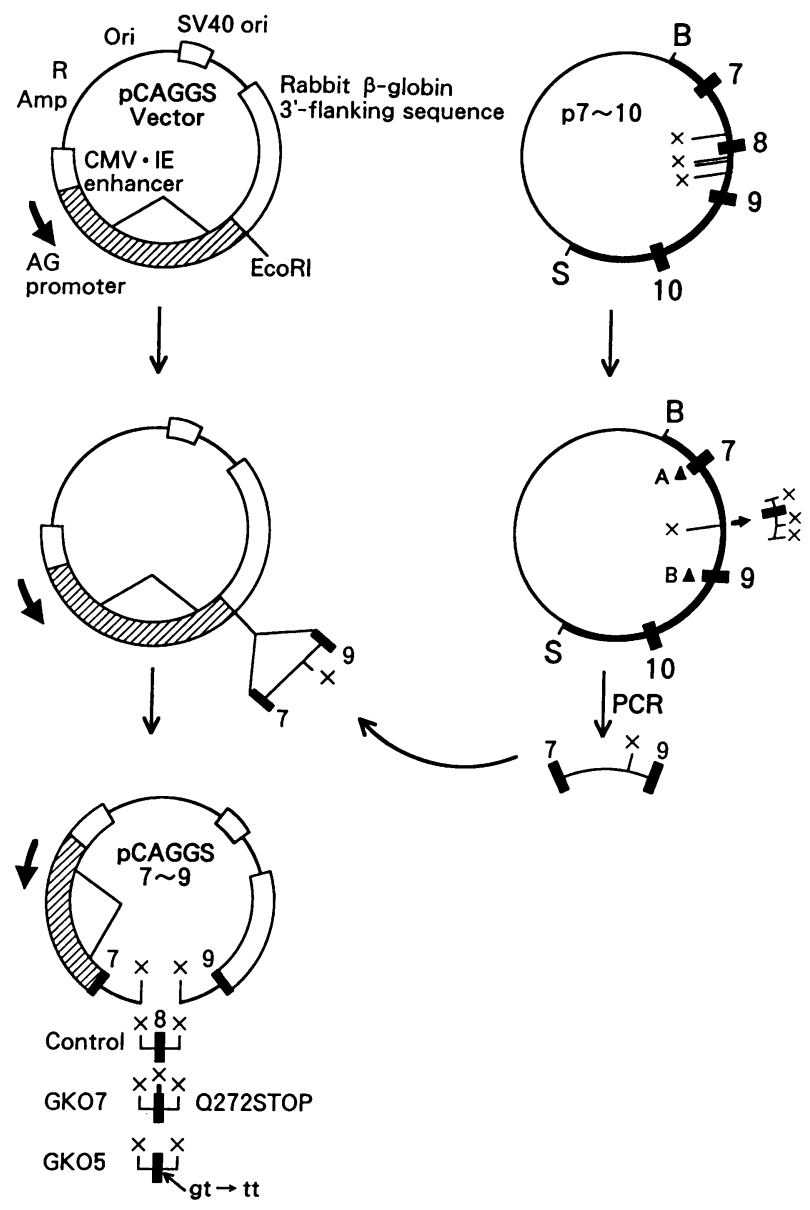

B
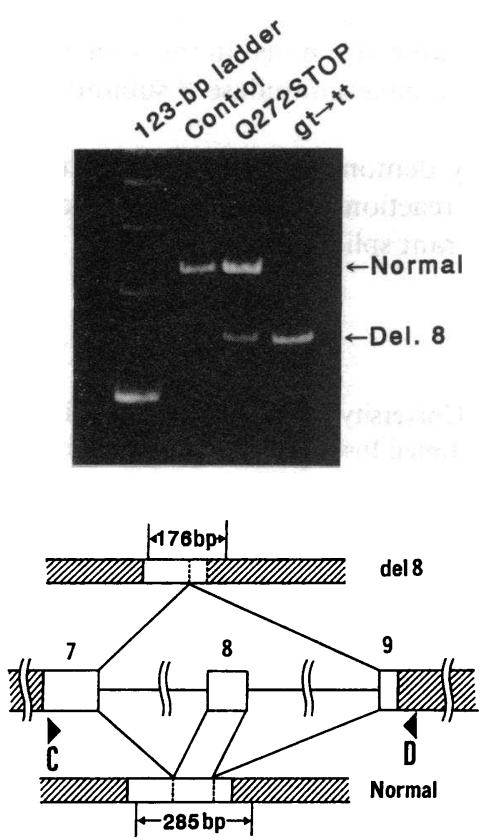

Figure 7. In vivo splicing experiments. $(A)$ Constructions of in vivo expression vectors. The upper left shows the pCAGGS expression vector (14). This vector transcribes well with an avian $\beta$-actin promoter ( $A G$ promoter). The p7-9 plasmid (upper right) includes $\mathrm{T} 2$ genomic sequence from a BamHI site of intron 6 to the middle of in- in her father. Normal sized cDNA fragment $\mathrm{C}$ from patient GK07 digested with Xbal followed by Southern blotting (Fig. 2 $D$ ) did not reveal an Xbal-digested fragment. Therefore, mRNA with the Q272STOP mutation was not detected in fibroblasts from GK07. The expression of the Q272STOP mutant allele apparently causes skipping of exon 8 .

Prediction of secondary structure. The Q272STOP mutation also lengthens two palindromic sequences, as indicated by the arrows in Fig. $5 \mathrm{~A}$. The predicted pre-mRNA secondary structure of the entire exon 8 sequence was then analyzed (Fig. 6 ). Residue $C$ at site -13 from the $5^{\prime}$ splice site in the normal sequence (Fig. $6 \mathrm{~A}$, encircled) seems to be important for the stem loop structure of the exon 8 sequence, because the $C$ to $T$ substitution in the mutant sequence resulted in a break in the predicted structure, the result being a local stem loop formation just upstream from the $5^{\prime}$ splice site (Fig. $6 \mathrm{~B}$ ). The predicted local stem loop may prevent the $5^{\prime}$ splicing site from forming a spliceosome.

In vivo splicing experiments. To verify that the $\mathrm{Q} 272 \mathrm{STOP}$ mutation is the cause of exon 8 skipping in GK07, in vivo splicing experiments were carried out. Fig. $7 A$ shows the strategy for construction of vectors to analyze splicing. We prepared three plasmids: pCAGGS7-9 control had the normal sequence; pCAGGS7-9Q272STOP had the mutant sequence with Q272STOP; and pCAGGS7-9gt $\rightarrow$ tt had the mutant sequence at the $5^{\prime}$ splice site of intron 8 , which we previously identified in GK05 (11). These constructs ensured that differences among the three vectors were only the mutations we wanted to examine. The three pCAGGS7-9 vectors were expected to express chimeric mRNAs with the $5^{\prime}$ half derived from the human $T 2$ sequence and the $3^{\prime}$ terminal half derived from the rabbit $\beta$-globin sequence (Fig. 7 B, lower panel).

The three pCAGGS7-9 vectors were transfected into both COS-7 cells and human fibroblasts. No significant difference between cell lines was noted in the pattern of amplified fragments. Fig. $7 B$ shows the results of transfection into human fibroblasts. In cells transfected with the pCAGGS7-9 control, a chimeric cDNA with the exon 8 sequence was amplified (con-

tron 10 , which we previously cloned from a normal individual (8). $X$ indicates $\mathrm{XbaI}$ sites. After deletion of Xbal fragments, an exon $7-$ exon 9 region was amplified with $A$ and $B$ primers and with VENT DNA polymerase (New England BioLabs Inc., Beverly, MA), then it was subcloned to the EcoRI site of the pCAGGS vector. Three XbaI fragments around exon 8 , including $140 \mathrm{bp}$ of 3 ' region of the intron 7 and $90 \mathrm{bp}$ of 5 ' region of the intron 8 , were prepared and subcloned into the vector: one had a normal sequence (pCAGGS7-9control), another had the Q272STOP mutation (pCAGGS7-9Q272STOP), and the other had the $\mathrm{gt} 10 \mathrm{tt}$ mutation at the $5^{\prime}$ splice site of intron 8 , which was derived from GK05's fragment, as described elsewhere (11) (pCAGGS7-9gt $\rightarrow \mathrm{tt}$ ). These XbaI fragments were sequenced, and no other mutations were detected. $(B)$ Detection of chimeric cDNAs derived from transfected pCAGGS7-9 constructs. $2 \mu \mathrm{g}$ of the three different pCAGGS7-9 constructs were transfected to $5 \times 10^{5}$ cells of human fibroblasts with TransfectAce (GIBCO BRL) as recommended by the manufacturer. At $48 \mathrm{~h}$ after transfection, RNA was extracted from cells using the AGPC method (36). First strand cDNA was transcribed with D primer from the rabbit $\beta$-globin sequence and then was amplified with $C$ primer on $T 2$ exon 7 sequence and the $\mathrm{D}$ primer. Amplified fragments were electrophoresed on a $10 \%$ polyacrylamide gel. Arrows indicate the predicted positions of the normally spliced fragments ( Normal) and the fragments lacking the exon 8 sequence ( $D e l .8)$. 
trol) while the cells transfected with pCAGGS7-9gt $\rightarrow$ tt had only a chimeric cDNA lacking exon 8 (lane $g t \rightarrow t t$ ). In the pCAGGS7-9Q272STOP-transfected cells (lane Q272STOP), the mRNA fragment lacking the exon 8 sequence was generated together with that retaining exon 8 . The amount of the former was estimated to be one-fourth that of the latter by densitometric analysis of products amplified with ${ }^{32} \mathrm{P}$-end-labeled primers. Thus, it was clear that the exonic mutation can cause exon 8 skipping.

The discrepancy in the amount of mRNA with exon 8 sequence and the Q272STOP mutation between GK07 fibroblasts and the transfected cells is attributed to the minigene construct pCAGGS7-9. In fibroblasts from patient GK07, mRNA with the Q272STOP was prematurely terminated, although mRNA with exon 8 skipping did retain the original reading frame. Premature termination itself causes a marked reduction in the amount of mRNA, except when the termination codon is located in the penultimate or the last exons $(30$, 31 ). Since exon 8 is not the penultimate or the last exon, premRNA with Q272STOP mutation was probably unstable, was degraded rapidly, and was undetectable. On the other hand, the minigene had no efficient initiation ATG codon and lacked an open reading frame. Hence, both minigene mRNAs with/ without exon 8 sequence were in the same state regarding premature termination. In the case of in vivo splicing experiments such nonfunctional mRNA are detectable because the mRNAs are overexpressed.

\section{Discussion}

In this paper, we describe the characterization of two novel mutations in patient GK07 with T2 deficiency. She was found to be a compound heterozygote who inherited the G379V mutant allele from her mother and the Q272STOP allele from her father. The most striking finding in this work is that the exonic single base substitution (Q272STOP) 13 bases upstream from the $5^{\prime}$ splice site of intron 8 resulted in the skipping of exon 8 .

In cases of aberrant splicing, the mutations have been identified predominantly in the consensus sequences of the $5^{\prime}$ donor $(\mathrm{G} / \mathrm{gtcag})$ and $3^{\prime}$ acceptor splice sites. We have also reported such cases of typical mutations in T2 deficiency (1113). We first wondered if the Q272STOP mutation alone would be responsible for the skipping of exon 8 . However, we observed allelic association between the exonic mutation and exon 8 skipping and could detect no other possible cause of the exon skipping. A role for exon sequences and structure in splice site selection has been suggested by several investigators (1418 ). Robberson et al. (18) proposed the model of the exon as the unit of assembly in splicing: multiple splicing factors initially recognize sequences at the 3 ' end of an intron, then the spliceosome assembly machinery searches precursor RNA sequences hunting for the $5^{\prime}$ splice site lying a short distance ( $<300$ bases) downstream of $3^{\prime}$ intron sequences through the exon. They term this step "exon definition." This model adequately explains most aberrant splicings (18). As shown in Fig. 6 , the predicted mRNA secondary structure was altered by the Q272STOP mutation and resulted in a local stem loop structure just upstream of $5^{\prime}$ splice site of intron 8 . The altered secondary structure may prevent the 5 ' splice site from assembling splicing factors. We observed previously that a GT to TT transversion at the $5^{\prime}$ donor splice site of intron 8 caused the skipping of exon 8 in case of GK05 (11). This observation indicates that there was probably no usable $5^{\prime}$ cryptic site located in the vicinity of the authentic site. Hence, exon skipping can occur if the 5' splice site is affected by the Q272STOP mutation. Thus, the single base exonic substitution was considered the cause of skipping of exon 8 .

We characterized the effect of the Q272STOP mutation on exon 8 splicing by comparing it with the 5 ' splice site mutation in in vivo splicing experiments. The control sequence resulted in no skipping of exon 8 . In the case of the $5^{\prime}$ splice site mutation, a complete skipping of exon 8 was evident as expected. On the other hand, the Q272STOP mutation caused a partial skipping, with about one-fourth of the mRNA carrying the deleted exon 8 sequence. The results suggest that exon definition was partially blocked by the Q272STOP mutation. We propose that alteration of the pre-mRNA secondary structure in the exon 8 sequence by the Q272STOP mutation reduced access of splicing machinery to the 5 ' splice site of intron 8 and caused exon 8 skipping in some transcripts.

To our knowledge, there are only two precedents of exonic mutation which may affect $5^{\prime}$ splice site selection. Ricketts et al. (32) identified two mutations in exon 9 of the bovine thyroglobulin gene that were associated with skipping of exon 9. One was a nonsense mutation and the other was a $C$ to $G$ mutation located at positions -28 and -21 , respectively, from the $5^{\prime}$ splice site of intron 9. The latter exonic mutation created a strong palindromic sequence (TGGGCTTGGAGAGCCCAA GAAAT/gt), which would result in an alteration of the premRNA secondary structure in this region. Steingrimsdottir et al. (33) analyzed many mutants at the human hypoxanthine guanine phosphoribosyltransferase gene and detected 34 mutations that resulted in aberrant splicing. Among them, a mutation located 13 nucleotides from the 5 ' splice site of intron 8 caused exon 8 skipping in $90 \%$ of hypoxanthine guanine phosphoribosyltransferase transcripts. They attributed partial exon skipping to alteration of secondary structures by this substitution. These cases are similar to ours. Exonic mutations which affect 3 ' splice site selection were also noted in the human episialin gene and the human $\beta$-hexosaminidase $\beta$ subunit gene $(34,35)$.

This study conclusively demonstrates the importance of exon sequences in splicing reactions; even a single nucleotide substitution can cause aberrant splicing.

\section{Acknowledgments}

We thank J. Miyazaki (The University of Tokyo) for providing the pCAGGS vector, T. Osumi (Himeji Institute of Technology) for constructive discussions, and $\mathrm{M}$. Ohara for helpful comments.

This study was supported in part by grants for the promotion of science research $(03265102,05454286$, and 05670666$)$ from The Ministry of Education, Science, and Culture of Japan, by a grant (5A-6-02) from the National Center of Neurology and Psychiatry of the Ministry of Health and Welfare, and by a grant for Pediatric Research (3A0104) from the Ministry of Health and Welfare, Japan.

\section{References}

1. Daum, R. S., P. H. Lamm, O. A. Mamer, and C. R. Scriver. 1971. A "new" disorder of isoleucine catabolism. Lancet. ii:1289-1290.

2. Daum, R. S., C. R. Scriver, O. A. Mamer, E. Devlin, P. Lamm, and H. Goldman. 1973. An inherited disorder of isoleucine catabolism causing accumulation of $\alpha$-methylacetoacetate and $\alpha$-methyl- $\beta$-hydroxybutyrate, and intermittent metabolic acidosis. Pediatr. Res. 7:149-160. 
3. Sweetman, L. 1989. Branched chain organic acidurias. In The Metabolic Basis of Inherited Disease. 6th ed. C. R. Scriver, A. R. Beaudet, W. S. Sly, and D. Valle, editors. McGraw-Hill Inc., New York. 791-819.

4. Iden, P., B. Middleton, B. H. Robinson, W. G. Sherwood, K. M. Gibson, L. Sweetman, and O. Sovik. 1990. 3-Oxothiolase activities and $\left[{ }^{14} \mathrm{C}\right]-2-$ methylbutanoic acid incorporation in cultured fibroblasts from 13 cases of suspected 3-oxothiolase deficiency. Pediatr. Res. 28:518-522.

5. Yamaguchi, S., T. Orii, N. Sakura, S. Miyazawa, and T. Hashimoto. 1988. Defect in biosynthesis of mitochondrial acetoacetyl-coenzyme A thiolase in cultured fibroblasts from a boy with 3-ketothiolase deficiency. J. Clin. Invest 81:813-817.

6. Nagasawa, H., S. Yamaguchi, T. Orii, R. B. H. Schutgens, L. Sweetman, and T. Hashimoto. 1989. Heterogeneity of defects in mitochondrial acetoacetylCoA thiolase biosynthesis in fibroblasts from four patients with 3-ketothiolase deficiency. Pediatr. Res. 26:145-149.

7. Fukao, T., S. Yamaguchi, M. Kano, T. Orii, Y. Fujiki, T. Osumi, and T. Hashimoto. 1990. Molecular cloning and sequence of the complementary DNA encoding human mitochondrial acetoacetyl-coenzyme $A$ thiolase and study of the variant enzymes in cultured fibroblasts from patients with 3-ketothiolase deficiency. J. Clin. Invest. 86:2086-2092.

8. Kano, M., T. Fukao, S. Yamaguchi, T. Orii, T. Osumi, and T. Hashimoto. 1991. Structure and expression of human mitochondrial acetoacetyl-CoA thiolase-encoding gene. Gene (Amst.). 109:285-290.

9. Masuno, M., M. Kano, T. Fukao, S. Yamaguchi, T. Osumi, T. Hashimoto, E. Takahashi, T. Hori, and T. Orii. 1992. Chromosome mapping of the human mitochondrial acetoacetyl-coenzyme A thiolase gene to $11 \mathrm{q} 22.3-\mathrm{q} 23.1$ by fluorescence in situ hybridization. Cytogenet. Cell Genet. 60:121-122.

10. Fukao, T., S. Yamaguchi, S. Tomatsu, T. Orii, G. Fraudienst-Egger, L. Schrod, T. Osumi, and T. Hashimoto. 1991. Evidence for structural mutation (347Ala to $\mathrm{Thr}$ ) in a German family with 3-ketothiolase deficiency. Biochem. Biophys. Res. Commun. 179:124-129.

11. Fukao, T., S. Yamaguchi, T. Orii, R. B. H. Schutgens, T. Osumi, and T. Hashimoto. 1992. Identification of three mutant alleles of the gene for mitochondrial acetoacetyl-coenzyme A thiolase. A complete analysis of two generations of a family with 3-ketothiolase deficiency. J. Clin. Invest. 89:474-479.

12. Fukao, T., S. Yamaguchi, T. Orii, T. Osumi, and T. Hashimoto. 1992. Molecular basis of 3-ketothiolase deficiency: identification of an AG to AC substitution at the splice acceptor site of intron 10 causing exon 11 skipping. Biochim. Biophys. Acta. 1139:184-188.

13. Fukao, T., S. Yamaguchi, C. R. Scriver, G. Dunbar, A. Wakazono, M. Kano, T. Orii, and T. Hashimoto. 1993. Molecular studies of mitochondrial acetoacetyl-coenzyme A thiolase deficiency in the two original families. Hum. Mutat. 2:214-220.

14. Eperon, L. P., J. P. Estibeiro, and I. C. Eperon. 1986. The role of nucleotide sequences in splice site selection in eukaryotic pre-messenger RNA. Nature (Lond.). 324:280-282.

15. Reed, R., and T. Maniatis. 1986. A role for exon sequences and splice-site selection. Cell. 46:681-690.

16. Mardon, H. J., G. Sebastio, and F. E. Baralle. 1987. A role for exon sequences in alternative splicing of the human fibronectin gene. Nucleic Acids Res. 15:7725-7733.

17. Somasekhar, M. B., and J. E. Mertz. 1985. Exon mutations that affect the choice of splice sites used in processing the SV40 late transcripts. Nucleic Acids Res. 13:5591-5609.

18. Robberson, B. L., G. J. Cote, and S. M. Berget. 1990. Exon definition may facilitate splice site selection in RNAs with multiple exons. Mol. Cell. Biol. 10:84-
19. Fukao, T., S. Yamaguchi, T. Orii, T. Osumi, and T. Hashimoto. 1992. Molecular basis of 3-ketothiolase deficiency: detection of gene mutations and expression of mutant $\mathrm{cDNAs}$ of mitochondrial acetoacetyl-CoA thiolase. $J$. Inherited Metab. Dis. 15:815-820.

20. Niwa, H., K. Yamamura, and J. Miyazaki. 1991. Efficient selection for high-expression transfectants with a novel eukaryotic vector. Gene (Amst.). 108:193-200

21. Zuker, M., and P. Stiegler. 1981. Optimal computer folding of large RNA sequences using thermodynamics and auxiliary information. Nucleic Acids Res. 9:133-148.

22. Efstratiadis, A., F. C. Katatos, and T. Maniatis. 1977. The primary structure of rabbit $\beta$-globin mRNA as determined from cloned DNA. Cell. 10:571585

23. Yu Yang, S., X. Ying He Yang, G. H. Louie, H. Schulz, and M. Elzinga. 1990. Nucleotide sequence of the $f$ adA gene. J. Biol. Chem. 265:10424-10429.

24. Peoples, O. P., S. Masamune, C. T. Walsh, and A. J. Sinskey. 1987. Biosynthetic thiolase from Zoogloea ramigera. J. Biol. Chem. 262:97-102.

25. Dequin, S., R. Gloeckler, C. J. Herbert, and F. Boutelet. 1988. Cloning, sequencing and analysis of the yeast $S$. Uvarum ERG10 gene encoding acetoacetyl CoA thiolase. Curr. Genet. 13:471-478.

26. Fukao, T., K. Kamijo, T. Osumi, Y. Fujiki, S. Yamaguchi, T. Orii, and T. Hashimoto. 1989. Molecular cloning and nucleotide sequence of cDNA encoding the entire precursor of rat mitochondrial acetoacetyl-CoA thiolase. J. Biochem. 106:197-204.

27. Arakawa, H., M. Takiguchi, A. Amaya, S. Nagata, H. Hayashi, and M. Mori. 1987. cDNA derived amino acid sequence of rat mitochondrial 3-oxoacylCoA thiolase with no transient presequence: structural relationship with peroxisomal isozyme. EMBO (Eur. Mol. Biol. Organ.) J. 6:1361-1366.

28. Hijikata, M., N. Ishii, H. Kagamiyama, T. Osumi, and T. Hashimoto. 1987. Structural analysis of cDNA for rat peroxisomal 3-ketoacyl-CoA thiolase. J. Biol. Chem. 262:8151-8158.

29. Fairbairn, L. J., and M. J. A. Tanner. 1989. Complete cDNA sequence of human foetal liver peroxisomal 3-oxoacyl-CoA thiolase. Nucleic Acids Res. 17:3588.

30. Urlaub, G., P. J. Mitchell, C. J. Ciudad, and L. A. Chasin. 1989. Nonsense mutations in dihydrofolate reductase gene after RNA processing. $\mathrm{Mol}$. Cell. Biol. 9:2868-2880

31. Cheng, J., M. Fogel-Petrovic, and L. E. Maquat. 1990. Translation to nea the distal end of the penultimate exon is required for normal levels of spliced triosephosphate isomerase mRNA. Mol. Cell. Biol. 10:5215-5225.

32. Ricketts, M. H., M. J. Simons, J. Parma, L. Mercken, Q. Dong, and G. Vassart. 1987. A nonsense mutation causes hereditary goiter in the Afrikande cattle and unmasks alternative splicing of thyroglobulin transcripts. Proc. Natl. Acad. Sci. USA. 84:3181-3184.

33. Steingrimsdottir, H., G. Rowley, G. Dorado, J. Cole, and A. R. Lehmann. 1992. Mutations which alter splicing in the human hypoxanthine-guanine phosphoribosyltransferase gene. Nucleic Acids Res. 20:1201-1208.

34. Ligtenberg, M. J. L., A. M. C. Gennissen, H. L. Vos, and J. Hilkens. 1991 A single nucleotide polymorphism in an exon dictates allele dependent differential splicing of episialin mRNA. Nucleic Acids Res. 19:297-301.

35. Wakamatsu, N., H. Kobayashi, T. Miyatake, and S. Tsuji. 1992. A novel exon mutation in the human $\beta$-hexosaminidase $\beta$ subunit gene affects $3^{\prime}$ splice site selection. J. Biol. Chem. 267:2406-2413.

36. Chomczynski, P., and N. Sacchi. 1987. Single-step method of RNA isolation by acid guanidinium thiocyanate-phenol-chloroform extraction. Anal. Biochem. 162:156-159. 